\title{
Inflammation in venovenous extracorporeal membrane oxygenation: villain or innocent bystander?
}

\author{
Aidan Burrell ${ }^{1,2}$, Thomas Müller ${ }^{3}$ \\ ${ }^{1}$ Department of Intensive Care, Alfred Hospital, Melbourne, Australia; ${ }^{2}$ Department of Epidemiology and Preventative Medicine, Monash \\ University, Melbourne, Australia; ${ }^{3}$ Department of Internal Medicine II, University Hospital Regensburg, Regensburg, Germany \\ Correspondence to: Thomas Müller. Department of Internal Medicine II, University Hospital Regensburg, Regensburg, Germany. \\ Email: thomas.mueller@ukr.de. \\ Provenance: This is an invited article commissioned by the Editorial Board Member Prof. Federico Pappalardo (Department of Anesthesia and \\ Intensive Care, Advanced Heart Failure and Mechanical Circulatory Support Program, San Raffaele Scientific Institute and San Raffaele Vita-Salute \\ University, Milan, Italy). \\ Response to: Datzmann T, Träger K. What about prognostic outcome parameters in patients with acute respiratory distress syndrome (ARDS) treated \\ with veno-venous extracorporeal membrane oxygenation (VV-ECMO)? J Thorac Dis 2018;10:S2057-61.
}

Submitted Jun 23, 2018. Accepted for publication Jul 04, 2018.

doi: $10.21037 /$ jtd.2018.07.20

View this article at: http://dx.doi.org/10.21037/jtd.2018.07.20

In 2017 the International ECMO Network (ECMONet) published a position paper highlighting key areas of research for extracorporeal membrane (ECMO) (1). Research into inflammation potentially impacts multiple parts of this agenda.

Inflammation is central to the pathogenesis of adult respiratory distress syndrome (ARDS), the major cause of severe respiratory failure requiring venovenous (VV) ECMO. Research on this topic will undoubtedly lead to improved understanding of the mechanisms of this disease. It may also lead to future drug targets which modulate or selectively reduce excessive inflammatory states. Inflammatory biomarkers may help clinicians to better select patients most likely to benefit from VV-ECMO and those who will not. Biomarkers also have a potential role as physiological targets for phase II clinical trials, given the challenges in completing phase III trials in ECMO using clinical endpoints (2).

In our prospective, observational study (3), we found cytokines interleukin 6 (IL-6), interleukin 8 (IL-8) and tumor necrosis factor (TNF-alpha) levels rapidly fell on the first day following initiation of VV ECMO in patients with severe refractory ARDS. The study methodology meant we were not able to define the precise cause of this decline, but concluded that less aggressive ventilation was likely a key factor. By the initiation of VV-ECMO, invasiveness of ventilation could rapidly be decreased, which was the major acute intervention. However, to show convincingly that this was the reason for the observed interleukin decrease, a control group without ECMO would be needed to exclude other modulations in treatment like hydrocortisone or antibiotics.

We also found that more aggressive ventilation prior to ECMO (positive end-expiratory pressure $>15 \mathrm{cmH}_{2} \mathrm{O}$ and driving pressure $>19 \mathrm{cmH}_{2} \mathrm{O}$ ) was associated with higher cytokine levels, which most likely is a consequence of severity of disease. And we could report that higher IL6, IL- 8 and TNF- $\alpha$ levels before and during ECMO were associated with an increased risk of death. Importantly, this study was not designed to develop new markers for prediction of survival on VV-ECMO. As we observed much interpatient variation in cytokine levels, we are skeptical that the concentration of cytokines alone would be a valuable predictive tool for these patients.

Datzmann et al. have explored the above issues in their thought provoking review (Datzmann, fournal of Thoracic Disease, 2018). In keeping with the ECMONet research agenda, they ask important questions about how research into inflammation may impact the care of VV-ECMO patients: why does the inflammation persist after the pathogen has been cleared? Is preservation of the endothelial barrier and glycocalyx a good target for 
the treatment of ARDS? What is the impact of different mechanical ventilation settings on cytokine levels? How can we improve patient selection during VV-ECMO? They have also highlighted some of the complexities of this research, such as how the inflammation response is modulated by other treatments including antibiotics, steroids and prone positioning.

Our report convincingly proved that the initiation of VV-ECMO is followed by a striking fall in cytokines, although we did not prove by which mechanism. Currently, there is a lot of discussion in the critical care community whether cytokine removal strategies will positively impact on outcome, and so far this discussion remains largely speculative. We dare to continue being skeptical. Are cytokines the reason for multi-organ failure, or are they more a marker for disease severity, or both? We believe our study shows that ECMO by itself probably reduces cytokine levels to a higher degree than the proposed cytokine filters.

Future prospective trials may be able to shed more light on these questions, and whether inflammation is indeed, villain or innocent bystander in VV-ECMO. In the meantime, Datzmann et al. have highlighted key areas and challenges for research on this topic, and also several potential clinical applications.

In a foot-note: in 2014 our study group reported potential markers of mortality in a cohort of 304 adult ARDS patients on ECMO (4). A logistic prediction model comprising age, immunocompromised state, artificial minute ventilation, hemoglobin concentration and preECMO serum lactate showed best mortality prediction in our patients (area under curve, AUC: 0.75). The importance of lactate is again described by Bonizzoli et al. (5). However, it has to be kept in mind while an AUC of 0.75 may suggest a prognosis, it must not serve to accept or decline a potentially life-saving therapy.

\section{Acknowledgements}

None.

\section{Footnote}

Conflicts of Interest: The authors have no conflicts of interest to declare.

\section{References}

1. Combes A, Brodie D, Chen YS, et al. The ICM research agenda on extracorporeal life support. Intensive Care Med 2017;43:1306-18.

2. Combes A, Hajage D, Capellier G, et al. Extracorporeal Membrane Oxygenation for Severe Acute Respiratory Distress Syndrome. N Engl J Med 2018;378:1965-75.

3. Burrell AJC, Lubnow M, Enger TB, et al. The impact of venovenous extracorporeal membrane oxygenation on cytokine levels in patients with severe acute respiratory distress syndrome: a prospective, observational study. Crit Care Resusc 2017;19:37-44.

4. Enger T, Philipp A, Videm V, et al. Prediction of mortality in adult patients with severe acute lung failure receiving veno-venous extracorporeal membrane oxygenation: a prospective observational study. Crit Care 2014;18:R67.

5. Bonizzoli M, Lazzeri C, Cianchi G, et al. Serial Lactate Measurements as a Prognostic Tool in Venovenous Extracorporeal Membrane Oxygenation Support. Ann Thorac Surg 2017;103:812-8.
Cite this article as: Burrell A, Müller T. Inflammation in venovenous extracorporeal membrane oxygenation: villain or innocent bystander? J Thorac Dis 2018;10(8):E652-E653. doi: 10.21037/jtd.2018.07.20 